\title{
Adiponectin normalization: a clue to the anti-metabolic syndrome action of rimonabant
}

\author{
Maynadier Marie , Basile Ilaria , Gary-Bobo Magali * \\ IRCM, Institut de Recherche en Cancérologie de Montpellier INSERM : U896, Université Montpellier I, CRLCC Val d'Aurelle - Paul \\ Lamarque, 208, rue des Apothicaires - 34298 Montpellier Cedex 5,FR \\ * Correspondence should be adressed to: Magali Gary-Bobo <magali.gary-bobo@valdorel.fnclcc.fr>
}

\begin{abstract}
Obesity, currently associated with metabolic syndrome is characterized by an excessive fat storage in different organs, in particular adipose tissue, inducing the loss of its structural and functional integrity. The awareness of the importance of adipose tissue endocrine function and the key role of adipocytokines such as adiponectin in obesity and metabolic syndrome display, the necessity to develop new drugs able to exert a specific action on adipose tissue and on adiponectin level. Rimonabant, an anti-obesity drug presents a dual effect by decreasing food intake and importantly increasing adiponectin. This review focusses on the key role of adiponectin regulation in the success of rimonabant and suggests that this adipohormone may be considered as a therapeutic target to design innovative and promising anti-obesity and antimetabolic syndrome drugs.
\end{abstract}

MESH Keywords Adiponectin ; metabolism ; Adipose Tissue ; drug effects ; metabolism ; Animals ; Anti-Obesity Agents ; pharmacology ; Body Fat Distribution ; Clinical Trials as Topic ; Drug Delivery Systems ; Humans ; Metabolic Syndrome X ; drug therapy ; physiopathology ; Obesity ; drug therapy ; physiopathology ; Piperidines ; pharmacology ; Pyrazoles ; pharmacology

Author Keywords obesity ; metabolic syndrome ; adipose tissue ; adiponectin ; rimonabant

\section{OBESITY AND ASSOCIATED METABOLIC SYNDROME: THE CURSE OF THE TWENTY-FIRST CENTURY}

Obesity is a world wide health problem particularly in rich and industrialized countries, which allow consumption devoid of limits and rules. Today obese people represent a third of Americans and a fifth of Europeans and this pandemic affects more children every year [1].

The seriousness of obesity depends on its associated metabolic disorders that strongly increase the risk of cardiovascular diseases [2]. Obesity is a metabolic disease currently associated with a cluster of chronic and progressive pathologies presenting several features of metabolic syndrome, including type 2 diabetes, hyperinsulinemia and insulin resistance, dyslipidemia, atherosclerosis, hypertension, steatohepatitis, inflammation and cancer [3,4]. The excessive fat accumulation in principal organs and tissues involved in energy metabolism regulation such as adipose tissue, liver and muscle, impairs tissue integrity and causes a confined inflammation characterized by an increase in pro-inflammatory cytokines such as tumor necrosis factor $\alpha$ (TNFa) [5]. This local inflammation may play a key role in the development of insulin resistance in these tissues. The increase in TNFa level could induce in its turn insulin resistance, probably by saturating the insulin signaling pathway [6,7]. Insulin resistance reduces fatty acid oxidation, markedly amplifies the pathological excessive fat storage and gives a chronic and progressive nature to this process. Obesity is also characterized by a whole inflammatory state [2] with an increase in circulating level of pro-inflammatory cytokines such as TNFa, C-reactive protein (CRP), interleukin-1- $\beta$ (IL-1 $\beta$ ), interleukine-6 (IL-6), plasminogen activator inhibitor 1 (PAI-1), transforming growth factor $\beta$ (TGF 3 ), and a decrease in anti-inflammatory cytokine levels such as adiponectin [8]. This obesity-associated inflammatory component seems to play an important role in the dramatic progression of obesity and metabolic syndrome [5]. In addition, metabolic dysfunction of principal organs involved in lipid and glucose metabolism is connected with a whole body lipid disturbance called dyslipidemia: characterized by an increase in circulating levels of cholesterol, triglycerides, and free fatty acids and by the reduction in the ratio of circulating levels of high density lipoprotein-cholesterol (HDLc) and low density lipoprotein-cholesterol (LDLc) [9]. Dyslipidemia is closely related to an increased risk of cardiovascular diseases including atherosclerosis, hypertension and myocardial infraction. The relationship between each main disorders and the metabolic syndrome is represented in Figure 1, where obesity, type 2 diabetes, dyslipidemia, inflammation and steatohepatitis supply this machinery leading to the increased risk of cardiovascular disorders.

Based on these observations, it is clear that structural and functional integrity of organs as the adipose tissue, involved in the regulation of lipid and glucose metabolism and in the maintenance of energy homeostasis, plays a crucial role in the pathophysiological processes of obesity and associated metabolic diseases clustered in the metabolic syndrome. The pharmacotherapy targeting the restoration of structural and functional integrity of metabolic organ as adipose tissue may be a promising pathway in the treatment of obesity and metabolic syndrome. This review highlights the involvement of adipose tissue integrity and adiponectin regulation in the success of anti-obesity and anti-metabolic syndrome drug as rimonabant.

\section{PATHOPHYSIOLOGICAL ROLE OF ADIPOSE TISSUE}


Adipose tissue has a highly specialized function of fat accumulation in situation of energy intake overload that can be used during periods of high energy consumption or food deprivation. This tissue is mainly composed by adipose cells (adipocytes) at different states of differentiation including adipoblastes, preadipocytes, immature and mature adipocytes [10,11]. Cellular distribution and relative abundance of each cellular subtype confer the heterogeneity characterizing this tissue, which is under hormonal and nutritional control. In pathological conditions, excessive adipose tissue development depends on hyperplasia (increase in the number of adipocytes by cell proliferation associated with recruitment of new adipose cells from precursors) and hypertrophy (increase in adipocyte size due to fat storage). Hypertrophy and hyperplasia induce a loss of adipose tissue heterogeneity associated with a dramatic disturbance to its structure and function [12].

This endocrine organ is able to express and secrete numerous molecules and biologically active proteins called adipocytokines or adipokines including leptin, TNFa, IL-6, IL-1 $\beta$, PAI-1, TGFß, angiotensinogen, adipsin, resistin, acylation stimulating protein (ASP) and adiponectin [13-15]. Through auto-, para- and endocrine mechanisms these adipocytokines (growth factors, hormones, cytokines) play multiple and important roles in several pathophysiological processes, particularly those involved in the regulation of energy metabolism and body weight homeostasis [16,17] but also in vascular, immune and reproductive systems [14]. The expression and secretion profile of these adipocytokines reflect the state of adipose tissue. The loss of adipose tissue heterogeneity is associated with the dysregulation in its endocrine function, enzyme activities and with the loss of its response to hormonal regulator such as insulin, leading to insulin resistance that plays a crucial role in the development and progression of obesity and associated metabolic diseases.

These observations suggest that therapeutic strategy leading to the restoration of adipose tissue integrity (structural heterogeneity and endocrine function) may be a promising pathway in obesity and metabolic syndrome treatment.

\section{ADIPONECTIN REGULATION AS A TARGET FOR OBESITY AND METABOLIC SYNDROME TREATMENT}

Adiponectin or adipocyte complement-related protein of $30 \mathrm{kDa}$ (Acrp30) also known as AdipoQ, ApM1 and GBP28 originally identified by four independent groups, is an adipocytokine exclusively expressed and secreted by adipose tissue [18-21]. It has been shown to regulate lipid and glucose metabolism and to play a key role in body weight regulation and homeostasis. Adiponectin has also been reported to be directly involved in several metabolic diseases such as obesity and associated complications including diabetes, hyperinsulinemia and insulin resistance, dyslipidemia, hypertension, hepatic pathologies and inflammation [22]. These pathologies represent principal features of metabolic syndrome which significantly increase the risk of cardiovascular diseases. Today, adiponectin is considered as a biomarker of metabolic syndrome and associated complications [23].

\section{Adiponectin and obesity, diabetes and insulin resistance}

Experimental and clinical studies have shown that adipose tissue expression and plasma levels of adiponectin are decreased in obese and type 2 diabetes subjects. The low plasma levels of adiponectin have been proposed to be a predictor for the development of insulin resistance and diabetes [24]. Furthermore in animal models of obesity and diabetes, adiponectin treatment reduced body weight, improved hyperglycemia, ameliorated hyperinsulinemia and insulin resistance and increased fatty acid oxidation and lipid clearance to significantly improved whole lipid profile [25-28]. These data confer to the adiponectin an important role in the regulation of lipid and glucose metabolism homeostasis as well as anti-obesity, anti-diabetes and insulin-sensitizing properties.

\section{Adiponectin and inflammation}

Metabolic diseases as obesity and diabetes are currently associated with a progressive low grade state of inflammation characterized by an increase in plasma and tissue (liver, muscle and adipose tissue) levels of pro-inflammatory cytokines [29]. Several studies in animal models and human, have reported that adiponectin plasma levels are inversely correlated with the increase in pro-inflammatory cytokines and markers such as C-reactive protein [30]. Moreover mice lacking adiponectin present a chronic inflammatory state associated to a strong increase in pro-inflammatory plasma levels, in particular the TNFa [31,32]. Treatment of these mice with adiponectin fully reverses this inflammation by reducing tissue and plasma levels of pro-inflammatory cytokines [33]. This anti-inflammatory activity of adiponectin may originate in the negative correlation between adiponectin and TNFa [31]. Adiponectin and TNFa antagonize each other in their target tissues, particularly in adipose tissue where the reduction of adiponectin expression is associated with the increase in pro-inflammatory cytokines underlining an inflammatory state of adipose tissue, which may progress and induce insulin resistance in this tissue [6,7]. Inflammation and insulin resistance in adipose tissue could lead to inflammation and insulin resistance in other organs as liver and muscle and to the appearance of serious pathologies as dyslipidemia, hepatic diseases and cardiovascular disorders [34].

\section{Adiponectin and steatohepatitis}

Hepatic steatosis, characterized by fat accumulation in liver, is a major metabolic complication associated with obesity and metabolic syndrome [35,36]. Steatohepatitis is the inflammatory state of this pathology characterized by an increase in tissue and plasma levels of pro-inflammatory cytokines such as TNFa, which is probably responsible for the progression to fibrosis and cirrhosis [33,36]. Fat 
accumulation in liver is reflected in a hepatic disorder in lipid and glucose metabolism, which could be due in large part to the increased TNFa levels and insulin resistance. The reduction of adiponectin plasma level is correlated to hepatic steatosis [23,37]. Adiponectin treatment of animal models abolish steatohepatitis by reducing hepatomegaly and fat accumulation in liver as well as plasma levels of liver feature markers such as amino-transferases [38]. Adiponectin could exerts its hepato-protective role by reducing TNFa hepatic and plasma levels [38,39], by reducing hyperinsulinemia and increasing insulin sensitivity of liver. In addition, adiponectin could improve steatohepatitis by inhibiting enzyme activity involved in fatty acid metabolism and by increasing free fatty acid oxidation in muscle and liver $[38,40]$.

\section{Adiponectin, dyslipidemia and cardiovascular diseases}

Overweight, obesity and associated metabolic disorders clustered in metabolic syndrome increase the risk of cardiovascular diseases development including hypertension, coronary atherosclerotic disease and congestive heart failure [41]. All these pathologies are closely associated with an atherogenic dyslipidemia that is a causal factor in cardiovascular diseases.

Dyslipidemia is characterized by elevated triglycerides, cholesterol, low high-density lipoprotein, and small dense low-density lipoprotein particles [42]. Circulating rate of adiponectin is inversely correlated to plasma levels of triglycerides, cholesterol and free fatty acids [43]. It is well established that dysregulation in adipocytokine levels may affect cardiovascular risk factors. The treatment with adiponectin reduces plasma levels of these parameters suggesting an important role of this hormone in lipid and glucose metabolism, and the involvement of its dysregulation on metabolic disorders associated with dyslipidemia [38]. This hormone induces the maintenance of functional integrity of endothelial cells and inhibits the proliferation of smooth muscle cells, notably in vascular remodeling processes [44]. Adiponectin also exerts an anti-hypertrophic action and protects against ischemic heart disease by a mechanism involving AMPK pathway [44]. In addition, a low adiponectin serum levels is strictly connected with hypertension, suggesting that hypoadiponectinemia could be considered as a predictor biomarker for cardiovascular diseases, while high levels of adiponectin are protective [45].

These findings confer to the adiponectin a cardio-protective role, which my be mediated by its anti-atherosclerosis, anti-thrombosis, anti-hypertension, anti-inflammatory and anti-dyslipidemic effects [44,45].

\section{OBVIOUSNESS OF A LINK BETWEEN CANNABINOID SYSTEM, OBESITY AND ASSOCIATED METABOLIC DISEASES}

Cannabinoid system, food intake and body weight

The delta-9-tetrahydrocannabinol $\left(\Delta^{9}\right.$-THC), the principal psycho-active component of marijuana or Cannabis Sativa, has been reported to stimulate food intake with a preference for palatable food high in fat and glucose [46]. The endogenous homologues of $\Delta^{9}$ -THC, called endocannabinoids: anandamide or arachidonoyl ethanolamide (AEA) and 2-arachidonoyl glycerol (2-AG) are lipidic neurotransmitters able to reproduce $\Delta^{9}$-THC-effects on food intake. These neurotransmitters (AEA and 2-AG) are produced in the principal satiety center (hypothalamus) and their levels are higher in obese than in lean animals. Synthetic cannabinoids (WIN 55,212-2 and CP 55,940) can also reproduce main effects of $\Delta^{9}$-THC and endocannabinoids in vitro and in vivo [46].

Cannabinoids (endogenous, exogenous and synthetic) exert their pharmacological activities after interaction with two receptors subtypes: type $1\left(\mathrm{CB}_{1}-\mathrm{R}\right)$ and type $2\left(\mathrm{CB}_{2}-\mathrm{R}\right)$ cannabinoid receptors [47]. $\mathrm{CB}_{1}-\mathrm{R}$ have been firstly described in hypothalamic neurons that expressed several neuropeptides involved in food intake and body weight regulation such as, neuropeptide $\mathrm{Y}$, cocaine amphetamine regulated transcription (CART), corticotropin-releasing hormone (CRH), melanin-concentrating hormone (MCH), and orexin [48].

SR141716 (rimonabant), a specific antagonist of $\mathrm{CB}_{1}-\mathrm{R}$, reduces food intake by blocking orexigenic effects of cannabinoids, probably by an antagonist activity on an endogenous cannabinoid tone in hypothalamus. In addition, knock-out mice for $\mathrm{CB}_{1}-\mathrm{R}\left(\mathrm{CB}_{1}-\mathrm{R} \mathrm{KO}\right)$ eat less and have a lower weight than their wild-type littermates [49].

We have reported that rimonabant reduced food intake and body weight in genetically obese Zucker (fa/fa) rats [50]. However, according to the time course of rimonabant-treatment, there is discordance between food intake and body weight reduction. Indeed, body weight loss induced by rimonabant comprises two phases. The early phase is principally food intake-regulation- dependent: during the first days of treatment, rimonabant reduced both food intake and body weight. This body weight loss induced by rimonabant probably originates from the decrease of food intake, which may be mediated by a mechanism involving the regulation of the expression and release of hypothalamic neuropeptides implicated both in the control of appetite and body-weight (i.e., a central effect of rimonabant) [49]. The second phase is food intakeregulation- independent: after the early phase (4 days of treatment) and throughout the entire period of treatment, rimonabant-induced body weight loss was maintained, whereas food intake increased. These observations suggest the involvement of metabolic and energy expenditure regulation in long term anti-obesity effects of rimonabant treatment as recently confirmed in human clinical trials [51]. 
Intraveinous injections of adiponectin induce body weight decrease in obese animals in a food intake independent manner [25] and effect of rimonabant on adiponectin mRNA expression in adipose tissue was investigated. We showed that rimonabant treatment stimulated adiponectin mRNA expression in adipose tissue of obese Zucker (fa/fa) rats. This stimulation was also found in cultured mouse adipocytes and was followed by an increase in adiponectin protein level. We then provided evidence that rat adipose tissue and cultured mouse adipocytes express $\mathrm{CB}_{1}-\mathrm{R}$. Importantly, adipose tissue of obese Zucker (fa/fa) rats over-expresses $\mathrm{CB}_{1}-\mathrm{R}$. We also shown that, rimonabant had no effect on adiponectin expression in adipose tissue of $\mathrm{CB}_{1}-\mathrm{R} \mathrm{KO}$ mice. These results demonstrated that rimonabant regulates adiponectin expression in adipocytes through a $\mathrm{CB}_{1}-\mathrm{R}$-mediated pathway. These results strongly suggest a role for adipose tissue $\mathrm{CB}_{1}-\mathrm{R}$ in the control of body weight homeostasis.

Hyperinsulinemia and insulin resistance are commonly associated with obesity and adiponectin plasma levels decrease [24-28]. We have shown that rimonabant treatment decreased the hyperinsulinemia characterizing obese Zucker (fa/fa) rats. This effect may be mediated at least in part by the up-regulation of adiponectin. The hyperinsulinemia reduction may participate to the overall anti-obesity actions of rimonabant.

Our data demonstrated that rimonabant regulated hormones implicated in lipid and glucose metabolism, by a mechanism involving a metabolic "peripheral" action in addition to its known "central" effect on food intake and revealed that adiponectin regulation probably played a key role in anti-obesity activities of rimonabant.

\section{$\mathrm{CB}_{1}-\mathrm{R}$ antagonism inhibits preadipocyte proliferation}

In obesity, the excessive fat storage induces marked changes in cellular composition and distribution of adipose tissue with alteration of its endocrine function. We have demonstrated that $\mathrm{CB}_{1}-\mathrm{R}$ blocking inhibited cell proliferation of cultured mouse preadipocytes accompanied by a rapid decrease in p42/44 MAP kinase activity [52]. Rimonabant acted also on endocrine function and enzyme content of cultured mouse preadipocytes, as shown by the increase in the expression of two late markers of adipocyte cell maturation such as adiponectin and glyceraldehyde-3- phosphate-dehydrogenase (GAPDH). Surprisingly, this regulation was not followed by morphological changes characteristic of adipocyte differentiation such as lipid accumulation, even if arrested adipocyte cell proliferation was generally followed by fat accumulation [13].

Our hypothesis is that, in this cellular model, rimonabant inhibits preadipocyte cell proliferation and induces an uncoupling of the association between the inhibition of proliferation and lipid accumulation. Although complementary research is needed to clarify this atypical property, the inhibition of preadipocyte cell proliferation and the induction of adipocyte late "maturation" marker without fat accumulation may participate in the anti-obesity effects of rimonabant, particularly in the reduction of fat mass and in the restoration of adipose tissue structure and endocrine function.

\section{$\mathrm{CB}_{1}-\mathrm{R}$ antagonism improves metabolic syndrome features}

Recently, we have displayed food intake-reduction independent effects of rimonabant on hepatic steatosis and associated-metabolic syndrome features (inflammation, dyslipidemia and low plasma levels of adiponectin) in obese Zucker (fa/fa) rats [53]. Our results showed that rimonabant-treatment reduced hepatomegaly, completely abolished hepatic steatosis (fatty liver) and decreased plasma levels of enzyme markers of liver damage (ALT, GGT and ALP). Interestingly, rimonabant-treatment strongly reduced elevated levels of hepatic TNFa that characterize the inflammatory state of fatty liver: steatohepatitis [54]. Elevated levels of hepatic pro-inflammatory cytokines, such as TNFa, were suggested to induce insulin resistance in liver and to be involved in the progression of steatohepatitis to hepatic fibrosis and cirrhosis [36,54]. Rimonabant also reduces elevated plasma levels of TNFa characterizing chronic systemic low-grade inflammation associated with obesity and metabolic diseases [55]. This reduction of plasma and hepatic TNFa levels by rimonabant is probably involved in the reversion of hepatic steatosis and may arrest or prevent the progression of steatohepatitis into fibrosis and cirrhosis and suggest an anti-inflammatory activity of rimonabant. These data reveal that rimonabant possesses a hepato-protective activity and suggest a new therapeutic role of this $\mathrm{CB}_{1}-\mathrm{R}$ antagonist in hepatic diseases [53,56]. Moreover, the steatohepatitis-resistance in $\mathrm{CB}_{1}-\mathrm{R}$ knock-out $\left(\mathrm{CB}_{1}-\mathrm{R} \mathrm{KO}\right)$ mice confirms the proposed role of endocannabinoid system in liver diseases [57].

Furthermore, we have reported that rimonabant-treatment improves dyslipidemia, a principal biochemical disorder leading to cardiovascular complications, by reducing plasma levels of cholesterol, free fatty acids and triglycerides, and importantly by increasing the HDLc/LDLc ratio [53]. These results, obtained in animal model, were also found in human clinical trials [58,59]. In addition, rimonabant-treatment of obese (fa/fa) rats increased and normalized plasma levels of adiponectin. So the improvement by rimonabant of obesity associated metabolic diseases could be due in a large part to the increase and the normalization of adiponectin and adipose tissue secretory function restoration and suggests a potential clinical application for this $\mathrm{CB}_{1}-\mathrm{R}$ antagonist in the treatment of liver diseases associated to obesity and metabolic syndrome.

\section{CONCLUSION}


All together these observations demonstrate that the loss of adipose tissue integrity appears as a major event leading to the development of metabolic disorders constituting metabolic syndrome. Indeed, hypertrophy and hyperplasia of adipose tissue is generally associated with insulin resistance and inflammation in this tissue and in others organs involved in energy metabolism such as muscle and liver. The overdevelopment of adipose tissue could also lead to fat accumulation in different organs engendering among others hepatic steatosis and dyslipidemia. In addition, the regulation of a hormone exclusively produced and secreted by adipose tissue such as adiponectin, seems to play a crucial role in obesity and associated diseases. Several studies have demonstrated that the success of rimonabant treatment was due in a large part to its peripheral effect at adipose tissue, and particularly to the regulation of adiponectin expression and plasma levels (Figure 2). The multi-protective effects of rimonabant may be mediated by an increase in protective cytokines or hormones such as adiponectin, which is able to improve disorders involved in the metabolic syndrome such as obesity, diabetes and insulin resistance, inflammation, steatohepatitis, dyslipidemia and cardiovascular disorders. Therefore, the development of new drugs exerting a specific action on adipose tissue to restore its integrity and regulate adiponectin level should be an original and a promising therapeutic axis to improve significantly parameters of metabolic syndrome.

\section{Footnotes:}

Teaser: This review describes the key role of adipose tissue integrity and more particularly the impact of adiponectin level on the success of an anti-obesity and anti-metabolic syndrome drug as rimonabant.

\section{References:}

- 1. Ogden CL 2007; The epidemiology of obesity. Gastroenterology. 132: 2087- 2102

- 2. Isomaa B 2003; A major health hazard: the metabolic syndrome. Life Sci. 73: 2395- 2411

- 3. Blaha MJ 2008; A practical "ABCDE" approach to the metabolic syndrome. Mayo Clin Proc. 83: 932- 941

- 4. Russo A 2008; Metabolic syndrome and cancer risk. Eur J Cancer. 44: 293- 297

- 5. Das UN 2001; Is obesity an inflammatory condition?. Nutrition. 17: 953- 966

- 6. Cheung AT 1998; An in vivo model for elucidation of the mechanism of tumor necrosis factor-alpha (TNF-alpha)-induced insulin resistance: evidence for differential regulation of insulin signaling by TNF-alpha. Endocrinology. 139: 4928- 4935

- 7. Hotamisligil GS 1994; Tumor necrosis factor alpha inhibits signaling from the insulin receptor. Proc Natl Acad Sci U S A. 91: 4854- 4858

- 8. Sharma AM , Chetty VT 2005; Obesity, hypertension and insulin resistance. Acta Diabetol. 42: (Suppl 1) S3- 8

- 9. Adiels M 2008; Fatty liver, insulin resistance, and dyslipidemia. Curr Diab Rep. 8: 60- 64

- 10. Chaldakov GN 2003; Adipobiology of disease: adipokines and adipokinetargeted pharmacology. Curr Pharm Des. 9: 1023- 1031

- 11. Avram AS 2005; Subcutaneous fat in normal and diseased states: 2. Anatomy and physiology of white and brown adipose tissue. J Am Acad Dermatol. 53: 671- 683

- 12. Hausman DB 2001; The biology of white adipocyte proliferation. Obes Rev. 2: 239- 254

- 13. Trayhurn P , Beattie JH 2001; Physiological role of adipose tissue: white adipose tissue as an endocrine and secretory organ. Proc Nutr Soc. 60: $329-339$

- 14. Guerre-Millo M 2002; Adipose tissue hormones. J Endocrinol Invest. 25: 855- 861

- 15. Miner JL 2004; The adipocyte as an endocrine cell. J Anim Sci. 82: 935- 941

- 16. Havel PJ 2002; Control of energy homeostasis and insulin action by adipocyte hormones: leptin, acylation stimulating protein, and adiponectin. Curr Opin Lipidol. 13: 5159

- 17. Spiegelman BM , Flier JS 2001; Obesity and the regulation of energy balance. Cell. 104: 531- 543

- 18. Scherer PE 1995; A novel serum protein similar to C1q, produced exclusively in adipocytes. J Biol Chem. 270: 26746- 26749

- 19. Hu E 1996; AdipoQ is a novel adipose-specific gene dysregulated in obesity. J Biol Chem. 271: 10697- 10703

- 20. Maeda K 1996; cDNA cloning and expression of a novel adipose specific collagen-like factor, apM1 (AdiPose Most abundant Gene transcript 1). Biochem Biophys Res Commun. 221: 286- 289

- 21. Nakano Y 1996; Isolation and characterization of GBP28, a novel gelatin-binding protein purified from human plasma. J Biochem. 120 : 803- 812

- 22. Lara-Castro C 2007; Adiponectin and the metabolic syndrome: mechanisms mediating risk for metabolic and cardiovascular disease. Curr Opin Lipidol. 18: 263- 270

- 23. Ryo M 2004; Adiponectin as a biomarker of the metabolic syndrome. Circ J. 68: 975- 981

- 24. Trujillo ME , Scherer PE 2005; Adiponectin--journey from an adipocyte secretory protein to biomarker of the metabolic syndrome. J Intern Med. 257: 167- 175

- 25. Fruebis J 2001; Proteolytic cleavage product of 30-kDa adipocyte complementrelated protein increases fatty acid oxidation in muscle and causes weight loss in mice. Proc Natl Acad Sci U S A. 98: 2005- 2010

- 26. Berg AH 2001; The adipocyte-secreted protein Acrp30 enhances hepatic insulin action. Nat Med. 7: 947- 953

- 27. Yamauchi T 2001; The fat-derived hormone adiponectin reverses insulin resistance associated with both lipoatrophy and obesity. Nat Med. 7: 941 - 946

- 28. Combs TP 2001; Endogenous glucose production is inhibited by the adiposederived protein Acrp30. J Clin Invest. 108: 1875- 1881

- 29. Trayhurn P, Wood IS 2005; Signalling role of adipose tissue: adipokines and inflammation in obesity. Biochem Soc Trans. 33: 1078- 1081

- 30. Ouchi N 2003; Reciprocal association of C-reactive protein with adiponectin in blood stream and adipose tissue. Circulation. 107: 671- 674

- 31. Maeda N 2002; Diet-induced insulin resistance in mice lacking adiponectin/ACRP30. Nat Med. 8: 731- 737

- 32. Kubota N 2002; Disruption of adiponectin causes insulin resistance and neointimal formation. J Biol Chem. 277: 25863- 25866

- 33. Yokota T 2000; Adiponectin, a new member of the family of soluble defense collagens, negatively regulates the growth of myelomonocytic progenitors and the functions of macrophages. Blood. 96: 1723- 1732

- 34. de Luca C , Olefsky JM 2008; Inflammation and insulin resistance. FEBS Lett. 582: 97- 105

- 35. Brunt EM 2004; Nonalcoholic steatohepatitis. Semin Liver Dis. 24: 3- 20

- 36. Bugianesi E 2004; Non-alcoholic fatty liver and insulin resistance: a cause-effect relationship?. Dig Liver Dis. 36: 165- 173

- 37. Hui JM 2004; Beyond insulin resistance in NASH: TNF-alpha or adiponectin?. Hepatology. 40: 46- 54

- 38. Xu A 2003; The fat-derived hormone adiponectin alleviates alcoholic and nonalcoholic fatty liver diseases in mice. J Clin Invest. 112: 91- 100

- 39. Masaki T 2004; Adiponectin protects LPS-induced liver injury through modulation of TNF-alpha in KK-Ay obese mice. Hepatology. 40: $177-184$

- 40. Chandran M 2003; Adiponectin: more than just another fat cell hormone?. Diabetes Care. 26: 2442- 2450

- 41. Bozkurt B , Deswal A 2005; Obesity as a prognostic factor in chronic symptomatic heart failure. Am Heart J. 150: 1233 - 1239

- 42. Grundy SM 2008; Metabolic syndrome pandemic. Arterioscler Thromb Vasc Biol. 28: 629- 636

- 43. Matsubara M 2002; Decreased plasma adiponectin concentrations in women with dyslipidemia. J Clin Endocrinol Metab. 87: 2764- 2769

- 44. Hopkins TA 2007; Adiponectin actions in the cardiovascular system. Cardiovasc Res. 74: 11- 18

- 45. Imatoh T 2008; Adiponectin levels associated with the development of hypertension: a prospective study. Hypertens Res. 31: 229- 233

- 46. Kogan NM , Mechoulam R 2006; The chemistry of endocannabinoids. J Endocrinol Invest. 29: 3- 14 
- 47. Pertwee RG 2008; The diverse CB1 and CB2 receptor pharmacology of three plant cannabinoids: delta9-tetrahydrocannabinol, cannabidiol and delta9tetrahydrocannabivarin. Br J Pharmacol. 153: 199- 215

- 48. Cota D 2003; The endogenous cannabinoid system affects energy balance via central orexigenic drive and peripheral lipogenesis. J Clin Invest. 112 : $423-431$

- 49. Di Marzo V 2001; Leptin-regulated endocannabinoids are involved in maintaining food intake. Nature. 410: 822- 825

- 50. Bensaid M 2003; The cannabinoid CB1 receptor antagonist SR141716 increases Acrp30 mRNA expression in adipose tissue of obese fa/fa rats and in cultured adipocyte cells. Mol Pharmacol. 63: 908- 914

- 51. Van Gaal L 2008; Efficacy and safety of rimonabant for improvement of multiple cardiometabolic risk factors in overweight/obese patients: pooled 1-year data from the Rimonabant in Obesity (RIO) program. Diabetes Care. 31: (Suppl 2) S229- 240

- 52. Gary-Bobo M 2006; The cannabinoid CB1 receptor antagonist rimonabant (SR141716) inhibits cell proliferation and increases markers of adipocyte maturation in cultured mouse 3T3 F442A preadipocytes. Mol Pharmacol. 69: 471- 478

- 53. Gary-Bobo M 2007; Rimonabant reduces obesity-associated hepatic steatosis and features of metabolic syndrome in obese Zucker fa/fa rats. Hepatology. 46 : $122-129$

- 54. Tilg H , Diehl AM 2000; Cytokines in alcoholic and nonalcoholic steatohepatitis. N Engl J Med. 343: 1467- 1476

- 55. Dandona P 2004; Inflammation: the link between insulin resistance, obesity and diabetes. Trends Immunol. 25: 4- 7

- 56. Teixeira-Clerc F 2006; CB1 cannabinoid receptor antagonism: a new strategy for the treatment of liver fibrosis. Nat Med. $12: 671-676$

- 57. Osei-Hyiaman D 2005; Endocannabinoid activation at hepatic CB1 receptors stimulates fatty acid synthesis and contributes to diet-induced obesity. J Clin Invest. 115: 1298- 1305

- 58. Despres JP 2005; Effects of rimonabant on metabolic risk factors in overweight patients with dyslipidemia. N Engl J Med. $353: 2121$ - 2134

- 59. Despres JP 2008; Efficacy and safety of the weight-loss drug rimonabant. Lancet. 371: 555-

\section{Figure 1}

Metabolic syndrome machinery. In this simplified scheme, obesity, type 2 diabetes, dyslipidemia, inflammation and steatohepatitis are represented as cycles that supply metabolic syndrome inducing the development of cardiovascular disorders (CVD). Each cycle of a metabolic disorder is induced by a main dysregulation such as, energy excess, hyperinsulinemia, cholesterol, free fatty acids (FFA) and triglycerides (TG), pro-inflammatory cytokines, fat and inflammation. Inside cycles, are enounced principal organs or tissues involved in this pathologies supplying metabolic syndrome: blood, AT (adipose tissue), liver and muscle. In addition, food intake decrease, energy expenditure increase and drugs acting on energy metabolism are represented as inhibitors of metabolic syndrome progression.

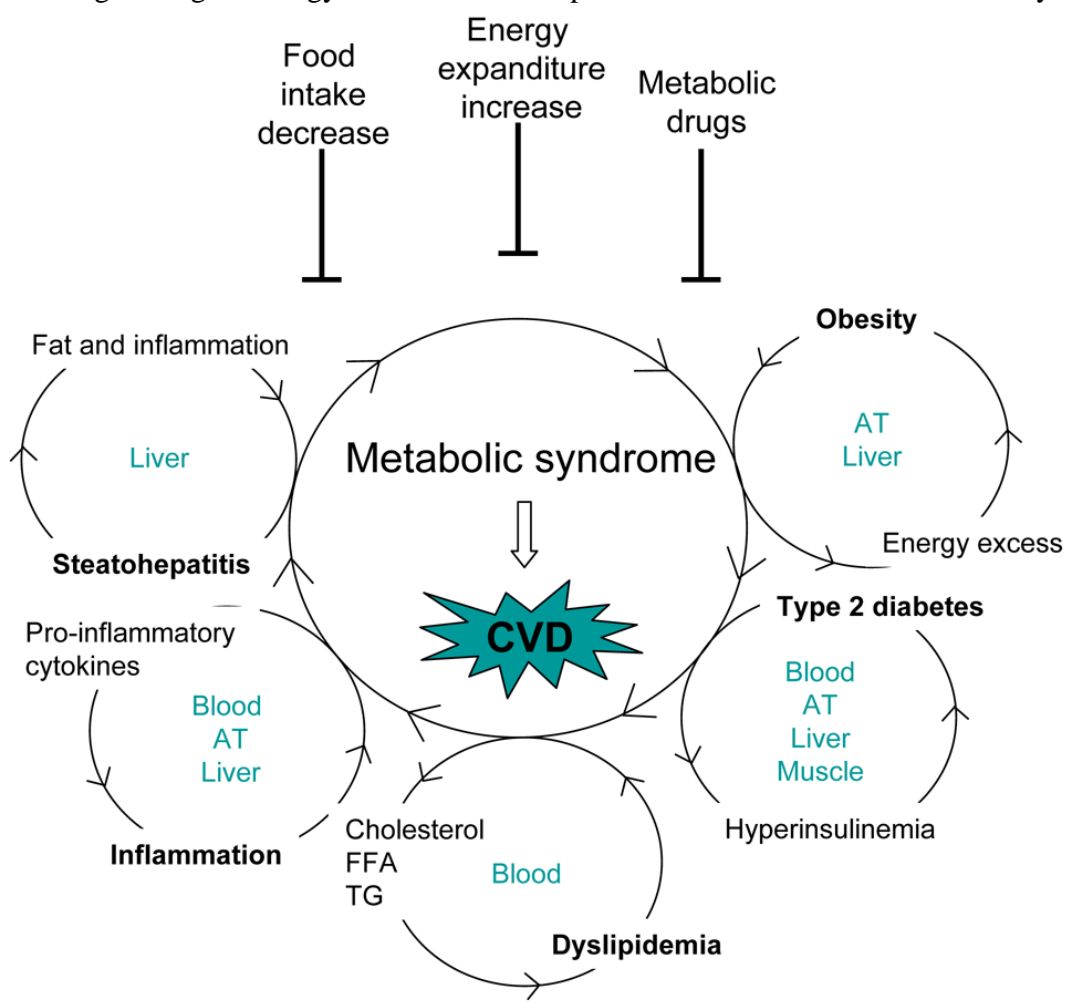


Figure 2

Anti-obesity peripheral action of rimonabant. By acting directly on type 1 cannabinoid receptors (CB1-R) of adipose tissue, rimonabant increases adiponectin level, which decreases: body weight and fat mass, hyperinsulinemia, dyslipidemia, inflammation and steatohepatitis. In addition adiponectin improves insulin sensitivity and glucose tolerance of several organ involved in lipid and glucose metabolism such as liver, adipose tissue and muscle that are at the origin of metabolic syndrome improvement inducing a decrease in cardiovascular risk factor.

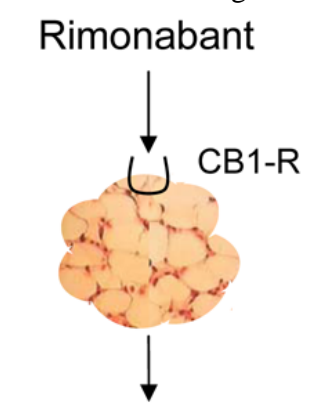

Adiponectin up-regulation

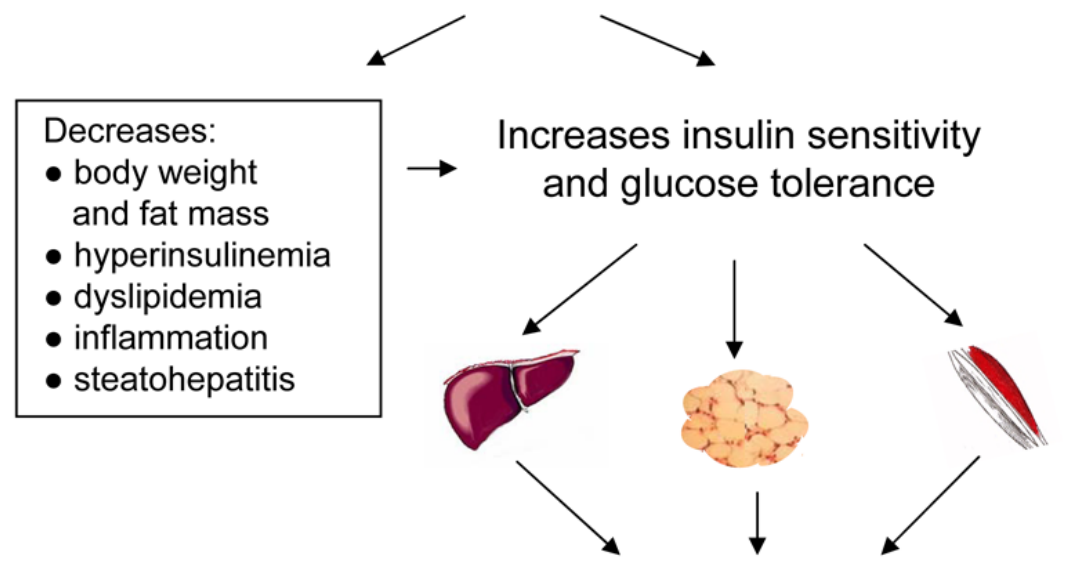

\section{Cardiovascular \\ Disorder Improvement}

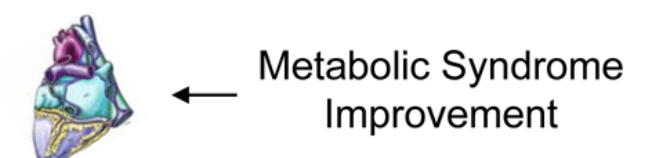

